\title{
Fatores associados à realização de citologia para prevenção de câncer do colo uterino em uma comunidade urbana de baixa renda
}

\author{
Factors associated with Pap smear for the prevention of \\ cervical cancer in a low income urban community
}

\author{
Germana Benevides Falcão1, Flávio Lúcio Pontes Ibiapina², Helvécio Neves Feitosa²,3, \\ Thiago Sant'Ana Feitosa², Patricia Dantas de Lacerda², José Ueleres Braga4, \\ Francisco Herlânio Costa Carvalho ${ }^{3,5}$
}

\begin{abstract}
Resumo
Objetivo: Avaliar a prevalência de realização da prevenção do câncer do colo uterino e fatores associados à coleta anual. Métodos: Estudo transversal conduzido entre agosto de 2010 e junho de 2011 por meio de entrevistas domiciliares com 144 mulheres de 25 a 52 anos em uma comunidade de baixa renda em Fortaleza (CE). Utilizou-se o teste do $\chi^{2}$ de Pearson e a análise de variância (ANOVA) na comparação entre os grupos que nunca realizaram coleta, realizaram não anualmente e aquelas que o fizeram com frequência anual. As variáveis com valores de $\mathrm{p}<0,20$ foram submetidas à regressão logística para estimativa da razão de chances (odds ratio - OR). Resultados: A proporção de mulheres que relatou coleta anual foi de 59,3\%. Trabalho remunerado $(p=0,04)$ e renda familiar $(p<0,01)$ mostraram associação significativa. Na regressão logística simples, o trabalho remunerado $(\mathrm{OR}=2,15)$, a classe econômica $(\mathrm{OR}=3,32)$ e a ocorrência de no máximo uma gestação $(\mathrm{OR}=2,73)$ e, na regressão logística múltipla, o trabalho remunerado $(\mathrm{OR}=2,63)$ e ocorrência no máximo de uma gestação $(\mathrm{OR}=2,60)$ mostraram-se associados à realização da coleta anual. Conclusão: Mulheres com trabalho remunerado e que tiveram no máximo uma gestação apresentaram uma chance maior de realização da citologia anual para prevenção do câncer de colo uterino. Palavras-chave: saúde da mulher; detecção precoce de câncer; câncer do colo do útero; citologia; programas de rastreamento.
\end{abstract}

\begin{abstract}
Objective: To evaluate the prevalence of prevention of cervical cancer and the factors associated with annual collection. Methods: Cross-sectional study conducted between August 2010 and June 2011 through interviews with 144 women 25-52 years old in a low income urban community in Fortaleza (CE). Were used the $\chi^{2}$ test and ANOVA comparing the groups never did collect, held annually and not those who made an annual basis. Variables with $p$ values $<0.20$ were subjected to logistic regression to estimate odds ratios (OR). Results: The proportion of women who reported annual collection was $59.3 \%$. Remunerated employment $(p=0.04)$, and family income $(p<0.01)$ showed significant association. In simple logistic regression, remunerated employment $(\mathrm{OR}=2.15)$, economic status $(\mathrm{OR}=3.32)$ and the having had the maximum of one pregnancy $(\mathrm{OR}=2.73)$ and, in multiple logistic regression, remunerated employment $(\mathrm{OR}=2.63)$ and the having had the maximum of one pregnancy $(\mathrm{OR}=2.60)$ were associated with annually periodicity for cervicovaginal smear. Conclusion: Women with remunerated employment and had no more than one pregnancy had a greater chance of achieving an annual frequency of cytology for preventing cervical cancer.
\end{abstract}

Keywords: women's health; early detection of cancer; uterine cervical neoplasms; cell biology; mass screening.

\footnotetext{
Trabalho realizado no Núcleo de Assistência Médica Integrada (NAMI) da Universidade de Fortaleza (UNIFOR) e no Departamento de Saúde Comunitária da Faculdade de Medicina da Universidade Federal do Ceará (UFC) - Fortaleza (CE), Brasil.

${ }^{1}$ Programa de Pós-Graduação em Saúde Pública da UFC - Fortaleza (CE), Brasil.

${ }^{2}$ Faculdade de Medicina da UNIFOR - Fortaleza (CE), Brasil.

${ }^{3}$ Departamento de Saúde Materno-Infantil da UFC - Fortaleza (CE), Brasil.

4Professor Adjunto da Universidade Estadual do Rio de Janeiro (UERJ) - Fortaleza (CE), Brasil.

${ }^{5}$ Departamento de Saúde Materno-Infantil e Departamento de Saúde Comunitária da UFC - Fortaleza (CE), Brasil.

Endereço para correspondência: Francisco Herlânio Costa Carvalho - Rua Professor Costa Mendes, 1.608, Bloco Didático, $5^{\circ}$ andar - Rodolfo Teófilo CEP: 60430-140 - Fortaleza (CE), Brasil - E-mail: herlaniocosta@uol.com.br

Fonte de financiamento: Programa de Pesquisas para o Sistema Único de Saúde (PPSUS), Ministério da Saúde (MS), Conselho Nacional de Desenvolvimento Científico e Tecnológico (CNPq), Fundação Cearense de Apoio ao Desenvolvimento Científico e Tecnológico (FUNCAP) e Secretaria de Saúde do Estado do Ceará (SESA) 02/2009. Conflito de interesses: nada a declarar.
} 


\section{INTRODUÇÃO}

O câncer do colo uterino é uma das neoplasias mais prevalentes em países de baixos e médios recursos. No Brasil, as taxas de incidência e mortalidade são consideradas intermediárias em relação aos demais países em desenvolvimento, porém altas se comparadas aos países desenvolvidos. Para 2012, o Instituto Nacional de Câncer (INCA) previu a ocorrência de 17.540 casos novos da neoplasia em todo o país, risco estimado de 17 casos para cada 100 mil mulheres ${ }^{1}$. Esses dados gerais não são capazes de demonstrar diferenças locorregionais e expor os verdadeiros indicadores entre as populações menos favorecidas. Estudos apontam associação do câncer cervical com o baixo nível socioeconômico e outras situações de vulnerabilidade das populações ${ }^{1,2}$.

A evolução de uma lesão inicial de colo para uma forma invasiva é lenta, podendo estender-se por até 20 anos. Essa característica torna a neoplasia maligna de controle efetivo por meio de rastreamento adequado. Programas de rastreio populacional sistemático e tratamento precoce das lesões precursoras podem reduzir em até $80 \%$ a mortalidade pelo câncer de colo. Para tanto, a cobertura mínima deve atingir $80 \%$ da população vulnerável e seguir protocolos preconizados ${ }^{2}$.

O Ministério da Saúde recomenda que o rastreamento seja feito por meio do exame citopatológico, a intervalos anuais, e após dois exames consecutivos negativos, a intervalos trienais. Para mulheres que já tiveram relação sexual, a coleta deve iniciar aos 25 anos e seguir até os 64 anos de idade 3 .

Nesta pesquisa, objetivou-se verificar a prevalência de realização da citologia cervicovaginal e fatores associados à coleta anual do exame em uma comunidade de baixa renda.

\section{METODOLOGIA}

Estudo transversal, de base populacional e caráter domiciliar realizado com uma população adstrita a uma área geográfica delimitada no Município de Fortaleza (CE), a Comunidade do Dendê. Trata-se de uma comunidade urbana de baixa renda caracterizada como aglomerado subnormal. Há comércios, creches, rádio comunitária, igrejas e delegacia, mas camuflam um labirinto de becos sem saída, onde a maioria dos moradores sobrevive em pequenos casebres.

Acredita-se que a comunidade teve origem no final da década de 1960, suas terras tendo sido invadidas e erraticamente ocupadas. Além da característica da ocupação de propriedade particular, há trechos invadidos em áreas de preservação ambiental, às margens do Rio Cocó. Os problemas da comunidade exacerbam-se frente ao contraste inerente ao local: a região é ladeada por bairros abastados da cidade, grandes avenidas, importantes centros comerciais e universidades particulares.

Realizou-se, previamente, georreferenciamento e cadastramento de todos os domićlios e moradores da comunidade. Uma equipe de visitadores, membros da própria comunidade, foi capacitada para abordagem dos moradores em domicílio, utilização do Sistema de Posicionamento Global (GPS) e correto registro dos imóveis. O cadastro domiciliar foi composto de endereço e caracterização do imóvel, membros da família, idade e sua posição familiar. Essa fase teve início em outubro de 2007 e foi concluída em abril de 2008. Os resultados principais desse cadastramento inicial de domicílios já foram publicados ${ }^{4}$.

O registro inicial constava de 10.892 moradores em 3.718 imóveis na comunidade. Observou-se um nível médio de aglomeração intradomiciliar. O número de moradores por domicílio foi de 4,3, em média. Entretanto, considerando o pequeno número de cômodos por residência, essa situação é considerada precária. Do total de moradores, 5.655 eram do sexo feminino e 2.605 encontravam-se entre 25 e 64 anos 4 .

Para o cálculo do tamanho da amostra, considerou-se que apenas 50\% realizam o exame preventivo anual para câncer de colo de útero ${ }^{5,6}$, entre aquelas que não tinham as exposições estudadas, e assumiu-se um erro $\alpha$ de $5 \%$ e um poder do estudo de $80 \%$. Para detectar uma razão de prevalência de 1,5 referente à associação entre os fatores estudados e a realização desse exame, seriam necessários 136 indivíduos. Pela possibilidade de perda de $20 \%$ da amostra selecionada, foram então sorteadas aleatoriamente 164 mulheres do registro inicial entre 25 e 64 anos, considerando a proporção de mulheres em cada uma das faixas etárias. Doze mulheres não foram encontradas em virtude de mudança de domicílio e 8 ainda não tinham iniciado vida sexual, perfazendo ao final uma amostra de 144 informantes.

As informações específicas para os objetivos deste estudo foram obtidas por meio de questionário estruturado com dois módulos (dados socioeconômicos e sobre a saúde da mulher). O questionário foi elaborado com base em instrumentos já validados, como aquele referente à classe econômica. Esse questionário foi avaliado na fase de pré-teste do Projeto Coorte Dendêt. A maioria das questões era fechada e aplicada diretamente à pessoa entrevistada, após assinatura do Termo de Consentimento Livre e Esclarecido. Os oito visitadores foram treinados para a aplicação das entrevistas e receberam manual completo com informações de orientação sobre o preenchimento. $\mathrm{O}$ trabalho de campo foi efetuado entre agosto de 2010 e junho de 2011.

A variável dependente foi definida como a realização da citologia cervicovaginal a intervalos anuais. As variáveis 
independentes foram: idade (estratificada por faixa etária: 25-39 e a partir de 40 anos); raça/cor da pele autorreferida (branca ou não branca); situação conjugal (com ou sem companheiro); escolaridade (estratificada por faixa: $\leq 4$, entre 5-8, $>8$ anos de estudo); trabalho remunerado; uso de cigarro; uso de bebida alcoólica; uso de drogas ilícitas; renda familiar (estratificada por faixas: menor que $\mathrm{R} \$ 800,00$ e maior ou igual a $\mathrm{R} \$ 800,00$ mensais - optou-se por esse ponto de corte por ser a renda média da população analisada, representando aproximadamente 1,5 salário mínimo vigente no país no período de estudo); escolaridade da pessoa de maior renda da família (estratificada por faixa $\leq 4$, entre 5-8, $>8$ anos de estudo); classe econômica (A1, A2, B1, B2, C1, C2, D e E, conforme Critério de Classificação Econômica Brasil Associação Brasileira de Empresas de Pesquisa - ABEP) ${ }^{7}$, dicotomizada em B1, B2, C1 e C2, D e E, para regressão logística; uso corrente ou prévio de métodos contraceptivos; idade da menarca (em anos); característica do ciclo menstrual (regular ou irregular); idade da primeira relação sexual (em anos); quantidade de parceiros sexuais; número de gestações; número e tipo de partos (parto vaginal ou cesariana); número de abortamentos; quantidade de filhos vivos; referência sobre doença sexualmente transmissível; doenças crônico-degenerativas referidas: diabetes, hipertensão, obesidade e dislipidemia; cirurgias já realizadas (especificação de cirurgia ginecológica ou não ginecológica, se houvesse). Para a regressão logística, optou-se também por dicotomizar as variáveis relacionadas à paridade em "se teve no máximo uma gestação", "não teve parto vaginal, nem cesárea", "teve algum aborto espontâneo ou provocado" e "tem filho vivo".

Os dados foram digitados em planilha do Excel e posteriormente analisados com a utilização do programa estatístico Stata ${ }^{\circ}$, versão 10.1 (StataCorp, USA).

A análise exploratória inicial estabeleceu correlações pelo teste do $\chi^{2}$ de Pearson, para as variáveis nominais, e pela análise de variância (ANOVA), para médias e desvios-padrões das variáveis contínuas. Para a avaliação inicial utilizou-se um nível de significância de até $20 \%$. Para expressar a magnitude das associações calcularam-se as razões de chance (odds ratio - OR) como expressões das razões de prevalência. Foram obtidas razões brutas $\left(\mathrm{OR}_{\text {bruta }}\right)$ das variáveis com $\mathrm{p}<0,20$ na avaliação inicial. Aquelas nas quais o intervalo de confiança não incluía a unidade foram levadas à regressão logística múltipla e apresentadas como $\mathrm{OR}_{\text {ajustada }}$ e intervalo de confiança de 95\% (IC95\%).

O protocolo de pesquisa foi submetido ao Comitê de Ética da Universidade de Fortaleza (UNIFOR), parecer no 150/2008, e aprovado em 02 de junho de 2008 sob registro no CEP 08-167 e CAAE 1550.0.000.037-08.

\section{RESULTADOS}

Na ocasião da entrevista, as 144 participantes tinham entre 25 e 52 anos, com maior frequência na faixa etária de 30 a 39 anos $(45,1 \%)$. Observou-se média de idade de $35,3(\mathrm{DP}=7,7)$ anos. A maioria das entrevistadas informou ser católica $(81,3 \%)$, autorreferiu-se como não branca $(69,4 \%)$ e com companheiro $(65,3 \%)$. Constatou-se média de escolaridade de $8,8(\mathrm{DP}=3,9)$ anos de estudo.

A maioria das entrevistadas não exercia trabalho remunerado $(56,4 \%)$. A renda familiar foi em média $R \$ 760,21$ $(\mathrm{DP}=378,40)$ e variou desde a informação de ausência de renda até $\mathrm{R} \$ 2.000,00$ mensais. A vasta maioria $(77,8 \%)$ pertencia às classes econômicas C2 e D. Quanto aos hábitos, 18,1\% eram fumantes e $36,8 \%$ referiram consumir bebida alcoólica, seja regular ou apenas socialmente.

Com relação às características ginecológicas e obstétricas, observou-se que a menarca ocorreu em média aos 12,9 ( $\mathrm{DP}=1,7)$ anos, com idade mínima aos 8 e máxima aos 17 anos. A maioria das mulheres $(62,5 \%)$ referiu ciclos menstruais regulares. A iniciação sexual deu-se em média aos 17,2 ( $\mathrm{DP}=3,3)$ anos, citados em média 2,4 parceiros por mulher. A maioria já havia engravidado (87,5\%), com média de 2,8 gestações e de 2,3 filhos vivos por mulher. Avaliando-se a ocorrência de doenças crônicas, $25,7 \%$ referiram alguma morbidade.

Em relação à realização do exame citopatológico para prevenção de câncer do colo uterino, 4 (2,8\%) entrevistadas informaram nunca ter realizado o preventivo, as outras 140 (97,2\%) referiram tê-lo feito pelo menos uma vez na vida. Maioria absoluta $(90,3 \%)$ relatou coletas periódicas dentro do intervalo trienal, sendo 59,3\% anualmente.

A análise exploratória inicial permitiu atentar para as diferenças entre os grupos de mulheres que nunca fizeram, fizeram, mas não anualmente ou fizeram anualmente a citologia para prevenção de câncer do colo uterino. A Tabela 1 mostra que a prevalência de realização da citologia anual foi significativamente mais elevada entre mulheres sem companheiro, de melhor classe econômica, que possuíam trabalho remunerado, com melhor nível de escolaridade e renda familiar acima de $R \$ 800,00$. As Tabelas 2 e 3 mostram, em dados, que não houve diferença com relação às características sexuais e reprodutivas.

Os resultados da regressão logística simples apontaram que o trabalho remunerado ( $\mathrm{OR}=2,15 ; \mathrm{IC} 95 \% 1,07-4,32)$, as classes econômicas mais favorecidas (OR=3,32; IC95\% 1,16-9,48) e o fato de ter tido no máximo uma gestação ( $\mathrm{OR}=2,73$; IC95\% 1,17-6,35) mostraram-se associados à periodicidade anual de realização da citologia. Na regressão logística múltipla, a classe econômica perdeu significância em virtude do intervalo de confiança desfavorável $\left(\mathrm{OR}_{\text {ajustada }}=2,02\right.$; IC95\% 0,95-4,27) (Tabela 4). 
Tabela 1. Características socioeconômicas e demográficas segundo realização da citologia para prevenção de câncer do colo uterino. Comunidade do Dendê, Fortaleza, 2010-2011

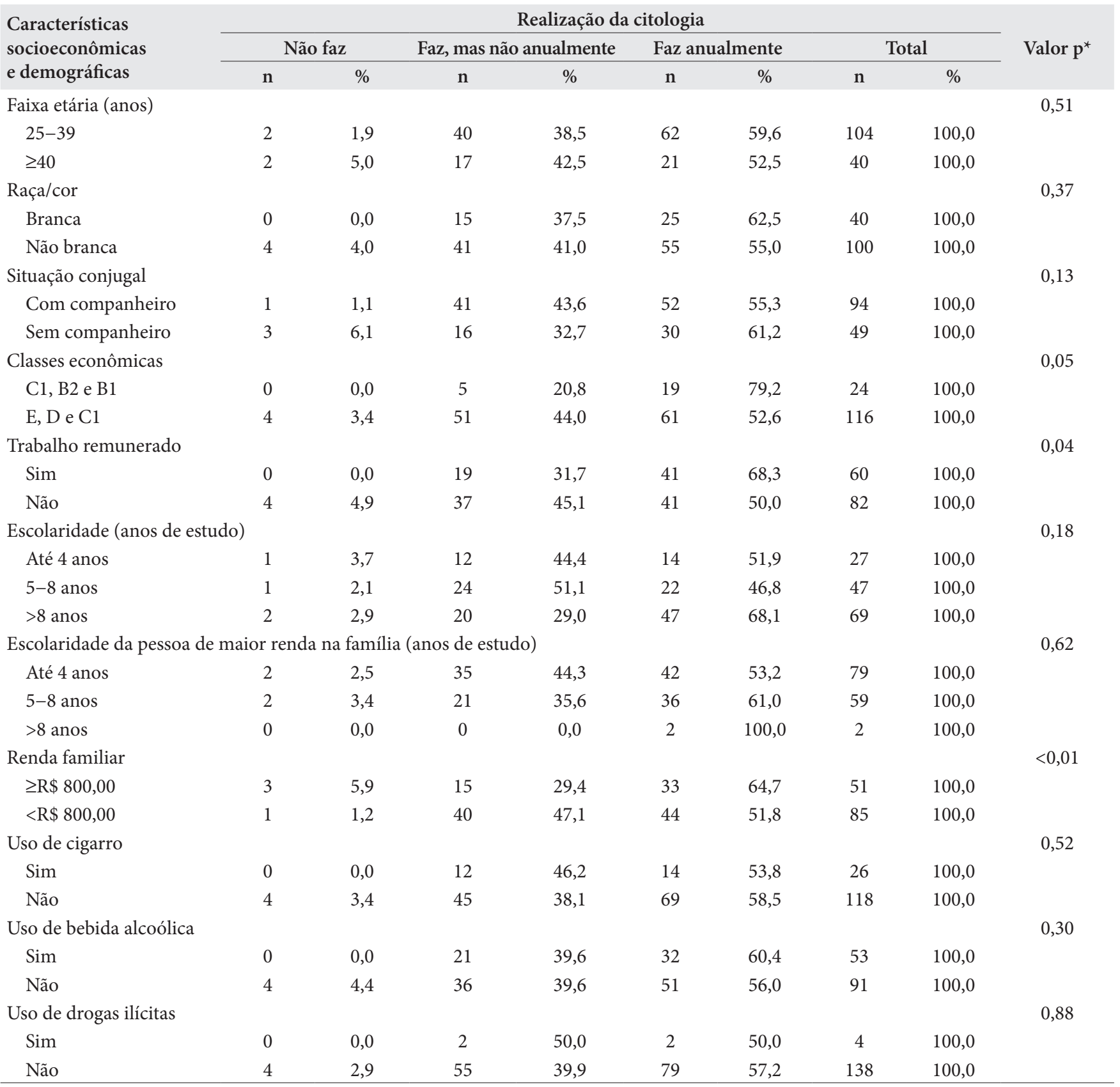

*Teste do $\chi^{2}$ de Pearson.

\section{DISCUSSÃO}

A quase totalidade $(97,2 \%)$ das mulheres entrevistadas referiu ter realizado o exame citológico para prevenção de neoplasia cervical pelo menos uma vez na vida, proporção ainda maior do que os $93,0 \%$ de cobertura evidenciados em Florianópolis (SC) ${ }^{8}, 82,4 \%$ em São Luís (MA) ${ }^{9}$ e $88,8 \%$ em Pelotas (RS) ${ }^{10}$.

Analisando a coleta do preventivo realizada a intervalos não superiores a 3 anos, a adesão ao exame também se mostrou elevada (90,3\%). Em duas capitais brasileiras,
Fortaleza e Rio de Janeiro, respectivamente 19,1\% e 16,5\% das mulheres tinham deixado de realizar a citologia no último triênio ${ }^{11}$. Outros estudos transversais, em Rio Branco $(\mathrm{AC})^{12}$ e São Leopoldo (RS) $)^{13}$, revelaram cobertura trienal para o preventivo de $85,5 \%$.

A cobertura expressiva encontrada na área de estudo é maior do que a de grandes cidades e capitais, incluindo a própria cidade onde a comunidade está localizada - Fortaleza. Uma das explicações para a boa adesão ao programa de rastreamento pode ser o fato de haver na comunidade uma Unidade 
Tabela 2. Características sexuais e reprodutivas (variáveis categóricas) segundo realização da citologia para prevenção de câncer do colo uterino. Comunidade do Dendê, Fortaleza, 2010-2011

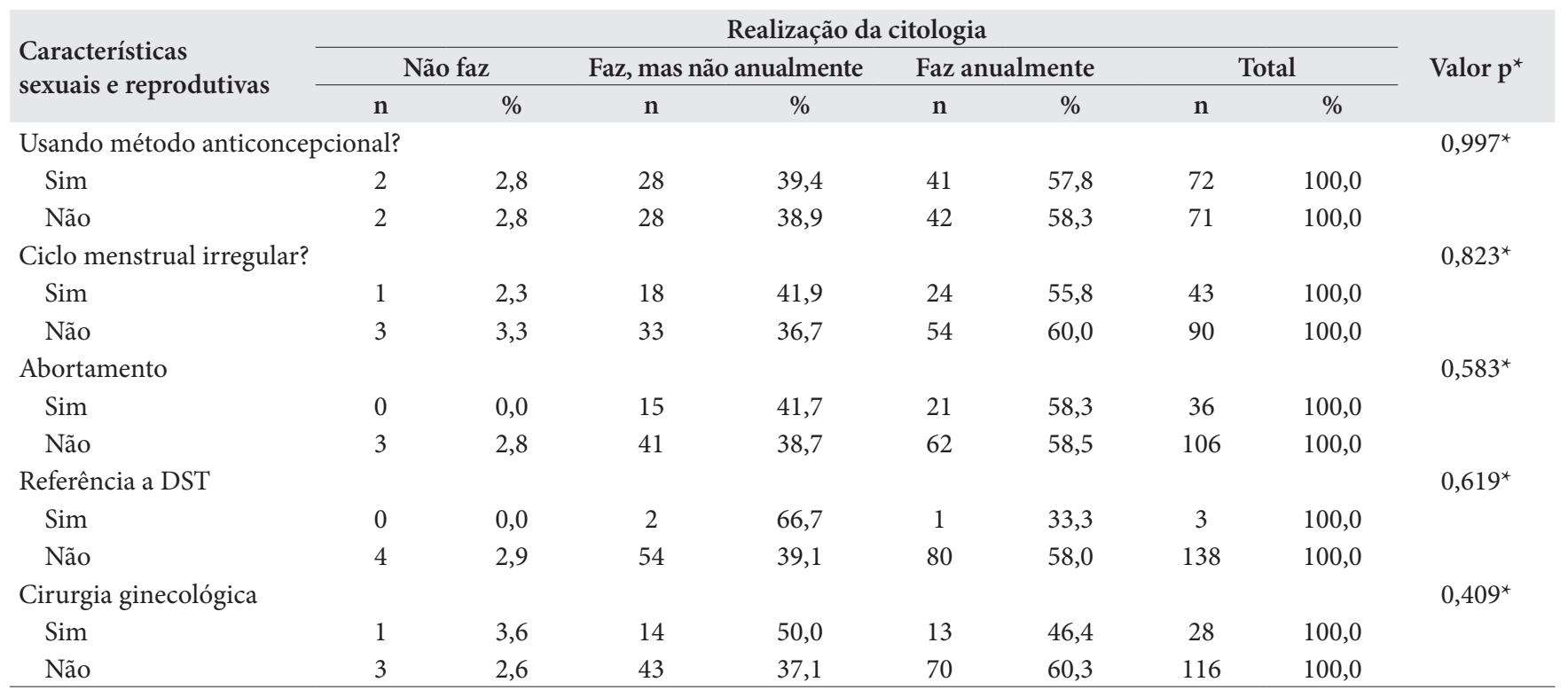

*Teste do $\chi^{2}$ de Pearson.

Tabela 3. Características sexuais e reprodutivas (variáveis contínuas) segundo realização da citologia para prevenção de câncer do colo uterino. Comunidade do Dendê, Fortaleza, 2010-2011

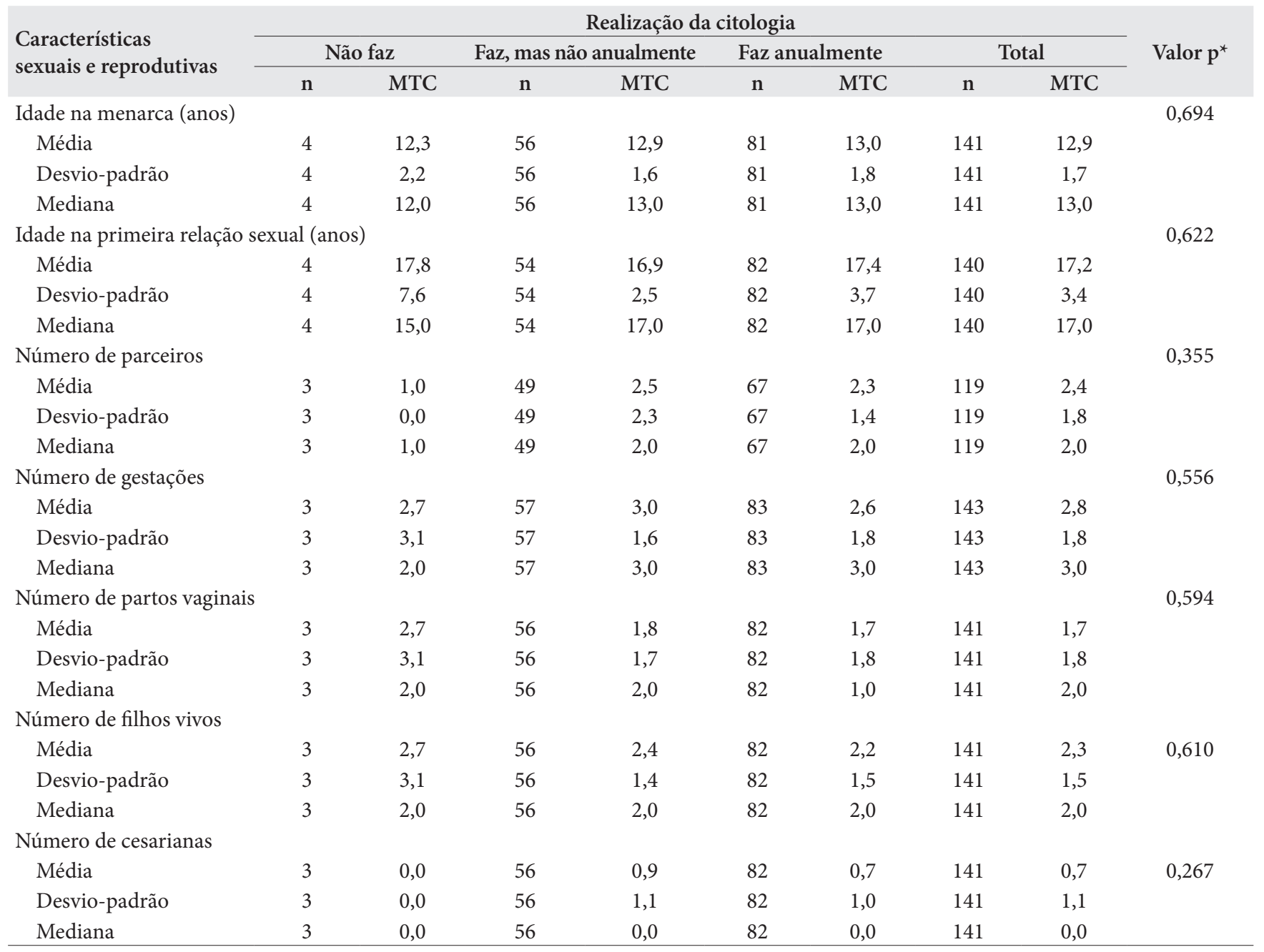

MTC: medida de tendência central; *ANOVA. 
Tabela 4. Características socioeconômicas, demográficas, sexuais e reprodutivas segundo regressão logística simples e múltipla para realização da citologia para prevenção de câncer do colo uterino com periodicidade anual. Comunidade do Dendê, Fortaleza, 2010-2011

\begin{tabular}{|c|c|c|c|c|c|}
\hline Fator & $\mathrm{n}$ & $\mathrm{OR}_{\text {bruta }}$ & IC95\% & $\mathrm{OR}_{\text {ajustada }}$ & IC95\% \\
\hline \multicolumn{6}{|l|}{ Situação conjugal } \\
\hline Com companheiro & 94 & 0,78 & $0,38-1,58$ & & \\
\hline Sem companheiro & 49 & 1 & & & \\
\hline \multicolumn{6}{|l|}{ Trabalho remunerado } \\
\hline Sim & 60 & 2,15 & $1,07-4,32$ & 2,63 & $1,49-4,64$ \\
\hline Não & 82 & 1 & & & \\
\hline \multicolumn{6}{|l|}{ Classes econômicas } \\
\hline $\mathrm{C} 1, \mathrm{~B} 2$ e B1 & 24 & 3,32 & $1,16-9,48$ & 2,02 & $0,95-4,27$ \\
\hline E, D e C2 & 116 & 1 & & & \\
\hline \multicolumn{6}{|c|}{ Escolaridade (anos de estudo) } \\
\hline$>8$ anos & 69 & 1,98 & $0,79-4,92$ & & \\
\hline $5-8$ anos & 47 & 0,81 & $0,31-2,10$ & & \\
\hline Até 4 anos & 27 & 1 & & & \\
\hline \multicolumn{6}{|c|}{ Renda familiar R\$ 800,00 } \\
\hline Sim & 51 & 1,70 & $0,83-3,49$ & & \\
\hline Não & 85 & 1 & & & \\
\hline \multicolumn{6}{|c|}{ Se teve no máximo 1 gestação } \\
\hline Sim & 36 & 2,73 & $1,17-6,35$ & 2,60 & $1,11-6,08$ \\
\hline Não & 107 & 1 & & 1 & \\
\hline \multicolumn{6}{|c|}{ Não teve parto vaginal, nem cesárea } \\
\hline Sim & 18 & 1,51 & $0,53-4,29$ & & \\
\hline Não & 123 & 1 & & & \\
\hline \multicolumn{6}{|c|}{ Teve algum aborto espontâneo ou provocado } \\
\hline Sim & 36 & 0,99 & $0,46-2,13$ & & \\
\hline Não & 106 & 1 & & & \\
\hline \multicolumn{6}{|l|}{ Tem filho vivo } \\
\hline Sim & 121 & 0,91 & $0,34-2,40$ & & \\
\hline Não & 20 & 1 & & & \\
\hline
\end{tabular}

Básica de Saúde (UBS) com equipes da Estratégia de Saúde da Família disponíveis, além de essa área ser de abrangência de uma instituição de ensino superior, com cursos de graduação (nove cursos na área da saúde) e pós-graduação.

Em conformidade com a recomendação do Ministério da Saúde, na qual a coleta da citologia deve ocorrer a intervalos anuais, sendo trienal quando 2 exames consecutivos são negativos, considerou-se de interesse verificar a cobertura anual $(59,3 \%)$ na Comunidade do Dendê, pois não se teve acesso aos resultados dos exames citológicos. Outros estudos brasileiros também evidenciaram cerca de 50\% das mulheres repetindo a coleta anualmente $e^{5,6}$.

A prevenção a intervalos anuais aponta para um grande contingente de exames desnecessários, sobrecarregando os serviços de saúde e onerando o sistema. Alguns autores vêm atentando para o caráter predominantemente oportunístico dessa coleta, considerando a necessidade de refrear exames em excesso, evitando repetições desnecessárias e coletas em pacientes jovens, fora da faixa preconizada ${ }^{6,14}$.
A maior parte das mulheres do estudo não exercia trabalho remunerado (57,4\%). Entre mulheres de baixa renda no Rio de Janeiro, a maioria não trabalhava $(52,2 \%)^{15}$. Dentre as $40,6 \%$ das mulheres que o exerciam, observou-se melhor prevalência de realização do exame preventivo (OR=2,63; IC95\% 1,49-4,64). Associações semelhantes foram evidenciadas em outras pesquisas ${ }^{12}$.

Tais observações remetem a discussões sobre paternalismo e desigualdade de gênero, tantas vezes repercutindo na autonomia feminina sobre o próprio corpo. A despeito de possuírem menor disponibilidade de tempo, pela própria situação ocupacional, as mulheres trabalhadoras mostraram-se significativamente mais assíduas à citologia oncótica, situação que pode ser entendida com base nas conquistas sociais e empoderamento por parte delas, conferindo-lhes maior autonomia e capacidade de escolha ${ }^{16}$. Todavia, deve-se levar em consideração que algumas empresas exigem exames periódicos dos funcionários, o que aumentaria as chances da realização de exames preventivos na rede assistencial pública ou na própria empresa. Nenhuma das pacientes referiu possuir acesso à rede suplementar de saúde. 
As mulheres do estudo que tiveram no máximo uma gestação também apresentaram maior chance de ter realizado o preventivo com periodicidade anual do que aquelas com duas ou mais gestações (OR=2,60; IC95\% 1,11-6,08). O período gestacional e o puerpério poderiam ser vistos como situações nas quais as mulheres têm maior chance de estar em contato com os serviços de saúde e, portanto, de realizar exames médicos. Entretanto, estudo realizado em município do Rio Grande do Sul constatou que o serviço local de saúde foi pouco efetivo em melhorar a adesão ao preventivo durante o pré-natal; revelando necessidade de melhor qualificação também na atenção à saúde durante a gestação, motivando e capacitando os profissionais para a importância da oportunização desse procedimento durante o pré-natal ${ }^{17}$.

Todavia, o que se vê na literatura clássica é a multiparidade como fator de risco para o aparecimento do câncer de colo uterino e estudos diversos associando a multiparidade à maior chance de não realização da citologia. Estudos tipo caso-controle verificaram associação de maior risco para desenvolvimento de leões do colo uterino ou não realização da citologia entre multíparas ${ }^{18}$.

No entanto, mesmo observando melhoria na cobertura nacional para citologia, o Brasil ainda tem altas taxas de mortalidade pela neoplasia e a maioria dos casos é detectada em estágio avançado ${ }^{19}$. Tal fato poderia ser explicado pela ineficiência dos programas de rastreio, visto que não estão sendo capazes de alcançar as mulheres de risco, as que nunca realizaram o exame ou o realizaram com periodicidade inadequada, além da necessidade de garantir seguimento e tratamento adequados aos casos detectados ${ }^{20}$.

Alta cobertura para prevenção de câncer de colo, com alta frequência de realização a intervalos curtos e fora da faixa preconizada, remete a indagações sobre a veracidade da coleta da citologia, quiçá confusão com exame ginecológico comum, e/ou sobre coletas desnecessárias, inseridas em um contexto de abordagem integral à saúde da mulher.
A importância recai sobre o rastreio organizado, para que haja efetividade nos programas adotados. A despeito da quantidade de coletas realizadas, as diferenças de impacto na incidência e mortalidade entre países desenvolvidos e em desenvolvimento são evidentes. Nestes, apesar da existência, em alguns deles, de programas de rastreamento, são em sua maioria oportunísticos e os exames são de qualidade discutível, realizados inadequadamente $e^{2,21}$.

Optou-se por inquérito domiciliar devido à maior representatividade da população de interesse. Buscar mulheres nas unidades de saúde, inevitavelmente, selecionaria um perfil diferenciado de amostragem em relação aos cuidados em saúde. No entanto, algumas outras informações não foram colhidas, como, por exemplo, dificuldades de acesso ao local da coleta, qualidade do atendimento prestado, compreensão das informações oferecidas que não foram alvo desta investigação.

Novos protocolos de pesquisa devem ser implementados, para avaliar quantitativa e/ou qualitativamente as dificuldades do sistema em oferecer um atendimento que gere impacto significativo nos indicadores de morbimortalidade. Informações sobre o papel de outros atores envolvidos (profissionais de saúde e gestores) também merecem ser investigadas.

\section{CONCLUSÃO}

Observou-se alta frequência de realização da citologia cervicovaginal para prevenção de câncer do colo uterino e alta prevalência de coletas anuais. $\mathrm{O}$ trabalho remunerado e o fato de ter tido no máximo uma gestação foram fatores relacionados à maior chance para realização do exame anualmente.

\section{AGRADECIMENTOS}

À Fundação Cearense de Apoio ao Desenvolvimento Científico e Tecnológico (FUNCAP), pelo apoio financeiro que viabilizou a realização deste projeto.

\section{REFERÊNCIAS}

1. Instituto Nacional de Câncer José Alencar Gomes da Silva. Coordenação Geral de Ações Estratégicas. Coordenação de Prevenção e Vigilância. Estimativa 2012: incidência de câncer no Brasil. Rio de Janeiro: INCA; 2011.

2. Sankaranarayanan R, Budukh AM, Rajkumar R. Effective screening programmes for cervical cancer in low- and middle-income developing countries. Bull World Health Organ. 2001;79(10):954-62.

3. Instituto Nacional de Câncer. Coordenação Geral de Ações Estratégicas. Divisão de Apoio à Rede de Atenção Oncológica. Diretrizes brasileiras para o rastreamento do câncer do colo do útero. Rio de Janeiro: INCA; 2011.
4. Moura KS, Bessa OAAC, Nuto SAS, Carmo Sá HL, Veras FMF, Braga JU. Projeto Coorte Dendê: diagnóstico demográfico e de moradia. Rev Bras Promoç Saúde. 2010;23(1):18-24.

5. Domingos ACP, Murata IMH, Pelloso SM, Schirmer J, Carvalho MDB. Câncer do colo do útero: comportamento preventivo de auto-cuidado à saúde. Ciênc Cuid Saúde. 2007;6(Supl. 2):397-403.

6. Vale DBAP, Morais SS, Pimenta AL, Zeferino LC. Avaliação do rastreamento do câncer do colo do útero na Estratégia Saúde da Família no Município de Amparo, São Paulo, Brasil. Cad Saúde Pública. 2010;26(2):383-390. 
7. Associação Brasileira de Empresas de Pesquisa [Internet]. Dados com base no Levantamento Sócio-econômico 2010 - IBOPE. São Paulo; 2012. [cited 2012 Feb 21] Available from: http://www.abep.org

8. Gasperin SI, Boing AF, Kupek E. Cobertura e fatores associados à realização do exame de detecção câncer de colo de útero em área urbana no Sul do Brasil: estudo de base populacional. Cad Saúde Pública. 2011;27(7):1312-22.

9. Oliveira MMHN, Silva AAM, Brito LMO, Coimbra LC. Cobertura e fatores associados a não realização do exame preventivo de Papanicolaou em São Luís, Maranhão. Rev Bras Epidemiol. 2006;9(3):325-34.

10. Dias-da-Costa JS, Olinto MTA, Gigante DP, Menezes AMB, Macedo S, Borba AT, et al. Cobertura do exame citopatológico na cidade de Pelotas, Rio Grande do Sul, Brasil. Cad Saúde Pública. 2003;19(1):191-7.

11. Martins LFL, Valente JG, Thuler LCS. Factors related to inadequate cervical cancer screening in two Brazilian states capitals. Rev Saúde Pública. 2009;43(2):318-25.

12. Borges MFSO, Dotto LMG, Koifman RJ, Cunha MA, Muniz PT Prevalência do exame preventivo de câncer do colo do útero em Rio Branco, Acre, Brasil, e fatores associados à não-realização do exame. Cad Saúde Pública. 2012;28(6):1156-66.

13. Muller DK, Dias-da-Costa JS, Luz AMH, Olinto MTA. Cobertura do exame citopatológico do colo do útero na cidade de São Leopoldo, Rio Grande do Sul, Brasil. Cad Saúde Pública. 2008;24(11):2511-20.

14. Dias MBK, Tomazelli JG, Assis M. Rastreamento do câncer de colo do útero no Brasil: análise de dados do Siscolo no período de 2002 a 2006. Epidemiol Serv Saúde. 2010;19(3):293-306.
15. Costa SH, Martins IR, Pinto CS, Freitas SRS. A prática de planejamento familiar em mulheres de baixa renda no município do Rio de Janeiro. Cad Saúde Pública. 1989;5(2):187-206.

16. Luszczynska A, Durawa AB, Scholz U, Knoll N. Empowerment beliefs and intention to uptake cervical cancer screening: three psychosocial mediating mechanisms. Women Health. 2012;52(2):162-81.

17. Gonçalves CV, Duarte G, Costa JSD, Quintana SM, Marcolin AC. Perdas de oportunidades na prevenção do câncer de colo uterino durante o prénatal. Ciênc Saúde Colet. 2011;16(5):2501-10.

18. Lima CA, Palmeira JAV, Cipolotti R. Fatores associados ao câncer do colo uterino em Propriá, Sergipe, Brasil. Cad Saúde Pública. 2006;22(10):2151-56.

19. Murta EFC, Franca HG, Carneiro MC, Caetano MSSG, Adad SJ, Souza MAH. Câncer do Colo Uterino: Correlação com o Início da atividade Sexual e Paridade. Rev Bras Ginecol Obstet. 1999;21(9):555-9.

20. Pinho AA, França Junior I, Schraiber LB, D’Oliveira, AFPL. Cobertura e motivos para a realização ou não do teste de Papanicolaou no Município de São Paulo. Cad Saúde Pública. 2003;19(Supl. 2):303-13.

21. Kjaer SK, Nielsen NH. Cancer of the female genital tract in circumpolar Inuit. Acta Oncol. 1996;35(5):581-7. 